\title{
Concanavalin A Enhanced Proliferation and Osteogenic Differentiation of Dental Pulp Stem Cells
}

\author{
Ketut Suardita ${ }^{1}$ Ira Arundina ${ }^{2}$ Udijanto Tedjosasongko ${ }^{3}$ Anita Yuliati ${ }^{4}$ Harry Huiz Peeters ${ }^{5}$ \\ I Komang Evan Wijaksana ${ }^{6}$ Meircurius Dwi Condro Surboyo ${ }^{7, \odot}$
}

\footnotetext{
1Department of Conservative Dentistry, Faculty of Dental Medicine, Universitas Airlangga, Jawa Timur, Indonesia

${ }^{2}$ Department of Oral Biology, Faculty of Dental Medicine,

Universitas Airlangga, Jawa Timur, Indonesia

${ }^{3}$ Department of Pediatric Dentistry, Faculty of Dental Medicine,

Universitas Airlangga, Jawa Timur, Indonesia

${ }^{4}$ Department of Dental Material, Faculty of Dental Medicine,

Universitas Airlangga, Jawa Timur, Indonesia

${ }^{5}$ Laser Research Center in Dentistry, Bandung, Indonesia

${ }^{6}$ Department of Periodontics, Faculty of Dental Medicine,

Universitas Airlangga, Jawa Timur, Indonesia

${ }^{7}$ Department of Oral Medicine, Faculty of Dental Medicine,

Universitas Airlangga, Indonesia
}

Eur J Dent 2020;14:123-127

\author{
Address for correspondence Udijanto Tedjosasongko, drg, Ph.D, \\ Sp. KGA(K), Department of Pediatric Dentistry, Faculty of Dental \\ Medicine, Universitas Airlangga. Jalan Prof. Dr. Moestopo 47 \\ Surabaya, 60132, Indonesia (e-mail: udijanto@fkg.unair.ac.id).
}

\begin{abstract}
Keywords

- concanavalin A

- dental pulp

- differentiation

- proliferation

- stem cell
\end{abstract}

Objective Dental pulp stem cells (DPSCs) can be used as a component in the formation of regenerative dentine during direct pulp capping therapy. Concanavalin $A(\operatorname{Con} A)$ is a type of lectin with a molecular weight of $26 \mathrm{kDa}$ derived from the Canavalia ensiformis plant. Lectins possess strong proliferation and differentiation abilities in various animal cells including lymphocytes, osteoblasts, and chondrocytes. The aim of study was to determine the effect of ConA on the proliferation and osteogenic differentiation of DPSCs in vitro.

Materials and Methods In this in vitro study, DPSCs were isolated from third molars before ConA induction was performed at concentrations of 5 and $10 \mu \mathrm{g} / \mathrm{mL}$. The proliferation assay was determined by 3-(4,5-dimethylthiazol-2-yl)-2, 5-diphenyltetrazolium bromide (MTT) assay. Osteogenic differentiation was determined by means of mineralization.

Statistical Analysis Data were analyzed using analysis of variance and a Student's $t$-test. The $p$-value was set at 0.05 .

Results The addition of 5 and $10 \mu \mathrm{g} / \mathrm{mL}$ of ConA to DPSCs can significantly increase the proliferation and osteogenic differentiation of DPSCs $(p \leq 0.05)$.

Conclusion ConA can increase the proliferation and osteogenic differentiation of DPSCs.

\section{Introduction}

Dental pulp stem cell (DPSC) is one of the promising dental stem cells for regenerative therapy. DPSCs show a higher proliferation rate compared with bone marrow mesenchymal stem cells (MSCs). In addition, DPSCs also have a stronger ability to differentiate into osteoblasts; therefore, DPSCs more appropriate for cell-based therapy. ${ }^{1}$ DPSCs are multipotent and can differentiate into chondrocytes, adipocytes, osteoblasts/osteocytes, myocytes, nerve cells, cardiomyocytes, and odontoblasts. ${ }^{2-4}$ DPSCs can be used to regenerate periodontal and dental tissue because they have the potential to form tissues such as bone, and are able to form dentin and pulp. ${ }^{2,5-7}$ With the ability to differentiate into odontoblast, DPSCs can 
be used as a component for regenerative dentine formation in direct pulp capping therapy.

The number of stem cells within the body is very limited. Consequently, the method of increasing their number in vitro without also affecting their differentiation must be developed. Several types of growth factors have been used to improve stem cell proliferation capability such as fibroblast growth factor-2 (FGF-2) or transforming growth factor- $\beta 1$ (TGF- $\beta 1){ }^{8}$ In addition, various factors are used to induce the differentiation capability of MSCs, for example, by using recombinant bone morphogenic protein-2 (BMP-2), which can increase the osteogenic differentiation of MSC. ${ }^{9}$ However, safer, cheaper, and more effective growth factors have yet to be identified.

In this study, concanavalin A (ConA) was investigated as a novel factor that may enhance the proliferation and osteogenic differentiation of DPSCs. ConA is a type of lectin with a molecular weight of $26 \mathrm{kDa}$ derived from the Canavalia ensiformis plant. Plant lectins play an important role in the cellular process. Lectins have strong proliferation and differentiation abilities in variety of animal cells, including lymphocytes, osteoblasts, and chondrocytes. ${ }^{10,11}$ The addition of ConA can enhance the process of mineralization or calcification of MSC derived from bone marrow. ConA can also increase levels of osteocalcin and BMP-2 proteins in MSC culture media. ${ }^{12}$ However, the effects of ConA on the proliferation and differentiation of DPSCs have yet to be investigated. The aim of this study was to determine the effect of ConA on the proliferation and osteogenic differentiation of DPSCs in vitro.

\section{Materials and Methods}

\section{DPSCs Isolation from the Pulp of Third Molars}

Ethical clearance for the research was obtained from the Health Research Ethical Clearance Commission, Faculty of Dental Medicine Universitas Airlangga (approval number 13/KKEPK.FKG/I/2016). In this in vitro study, dental pulp was extracted from the impaction vital teeth of healthy adults. Following extraction, teeth were placed into sterile phosphate-buffered saline (PBS; Sigma-Aldrich, Missouri, United States) solution. The teeth were sectioned axially at the cemento-enamel junction (CEJ) using a diamond rotary disc, and the dental pulp was removed. The pulp tissue was rinsed in $\alpha$-minimum essential medium ( $\alpha$-MEM) supplemented with $10 \%$ fetal bovine serum (FBS; Sigma-Aldrich) and antibiotics (100 units/mL penicillin $\mathrm{G}$ and $100 \mu \mathrm{g} / \mathrm{mL}$ streptomycin; Invitrogen Life Technologies; Carlsbad, California, United States) and minced into fragments of 1 to $2 \mathrm{~mm}^{3}$ before being placed in a $37^{\circ} \mathrm{C}$ humidified tissue culture incubator at $5 \% \mathrm{CO}_{2}$ for 4 days. The culture medium was changed every 3 days. When reaching $80 \%$ confluence, cells were harvested by using $0.05 \%$ trypsin-EDTA solution (Sigma-Aldrich) and subculture being performed for the experiments. DPSCs between passage 2 and 4 were used for the experiments. ${ }^{13}$ To prove that the cells obtained were MSC, CD105, and CD45 expression were examined. ${ }^{14}$

\section{Examination of DPSCs Proliferation Ability}

$4 \times 10^{4}$ DPSCs was cultured in 96-well tissue culture using $\alpha$-MEM medium, 10\% FBS (Sigma-Aldrich) and antibiotics (100 units/mL penicillin $\mathrm{G}$ and $100 \mu \mathrm{g} / \mathrm{mL}$ streptomycin). Five $\mathrm{ug} / \mathrm{ml}$ and $10 \mu \mathrm{g} / \mathrm{mL}$ of ConA were added in the treatment group for 24 hours. However, ConA was not added to the control group culture. After 48 hours of culture, a 3-(4,5-dimethylthiazol-2-yl)-2, 5-diphenyltetrazolium bromide (MTT) test was performed. $0.5 \mathrm{mg} / \mathrm{mL}$ of MTT was added to each well and incubated for 4 hours at $37^{\circ} \mathrm{C}$ and $5 \% \mathrm{CO}_{2}$. Dimethyl sulfoxide was added to each well and optical density (OD) was examined using an enzyme-linked immunosorbent assay reader (Bio-Rad; California, United States) at a wavelength of $550 \mathrm{~nm} .{ }^{15}$

\section{Examination of DPSCs Osteogenic Differentiation Ability}

A total of $1 \times 10^{5}$ DPSCs was cultured in 24-well tissue culture using Dulbecco's Modified Eagle Medium (DMEM) medium plus $10 \%$ FBS and antibiotics. After confluence, the medium was replaced with DMEM plus $10 \%$ FBS, $10 \mathrm{mM}$-glycerophosphate, $100 \mathrm{nM}$ dexamethasone, and $50 \mu \mathrm{g} / \mathrm{mL}$ ascorbic acid-2-phosphate (osteogenic medium). Five $\mu \mathrm{g} / \mathrm{ml}$ and $10 \mu \mathrm{g} / \mathrm{mL}$ of ConA were added to the treatment group, but not to the control group. The cells were incubated at $37^{\circ} \mathrm{C}$ in $5 \% \mathrm{CO}_{2}$. After 28 days, the culture medium was aspirated and the cell layer washed twice with PBS, and fixed with $95 \%$ ethanol for 10 minutes at room temperature. They were then washed twice with distilled water and stained for 30 minutes with $1 \%$ alizarin red S solution (Sigma-Aldrich; Merck KGaA) at room temperature. Photographs were taken after the cell layer had been washed with water using Canon Power Shot G12 digital camera (Tokyo, Japan). ${ }^{15}$

\section{Data Analysis}

Data were analyzed by means of a two-way analysis of variance (ANOVA) and expressed as mean \pm standard deviation. The statistical differences between the two groups were evaluated using a Student's $t$-test. In all the analyses, $p<0.05$ was considered to indicate a statistically significant difference.

\section{Results}

\section{DPSCs Isolation from the Pulp of Third Molars}

DPSCs appeared to be morphologically fibroblastic and after changing the culture media every 3 days, the number of DPSCs increased to the extent that on day 7 they appeared confluent ( - Fig. 1A). To ensure that the cells were stem cells, CD 105 and CD 45 expression was examined. DPSC expresses CD 105 but not CD 45 ( - Fig. 1B).

\section{DPSCs Proliferation Ability after Addition of ConA}

The effect of adding ConA to the proliferation of DPSCs was analyzed by MTT assay. The addition of 5 and $10 \mu \mathrm{g} / \mathrm{mL}$ of ConA can significantly increase the proliferation of DPSCs $(p \leq 0.05)$. A concentration of $10 \mu \mathrm{g} / \mathrm{mL}$ produced higher pro-

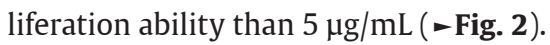



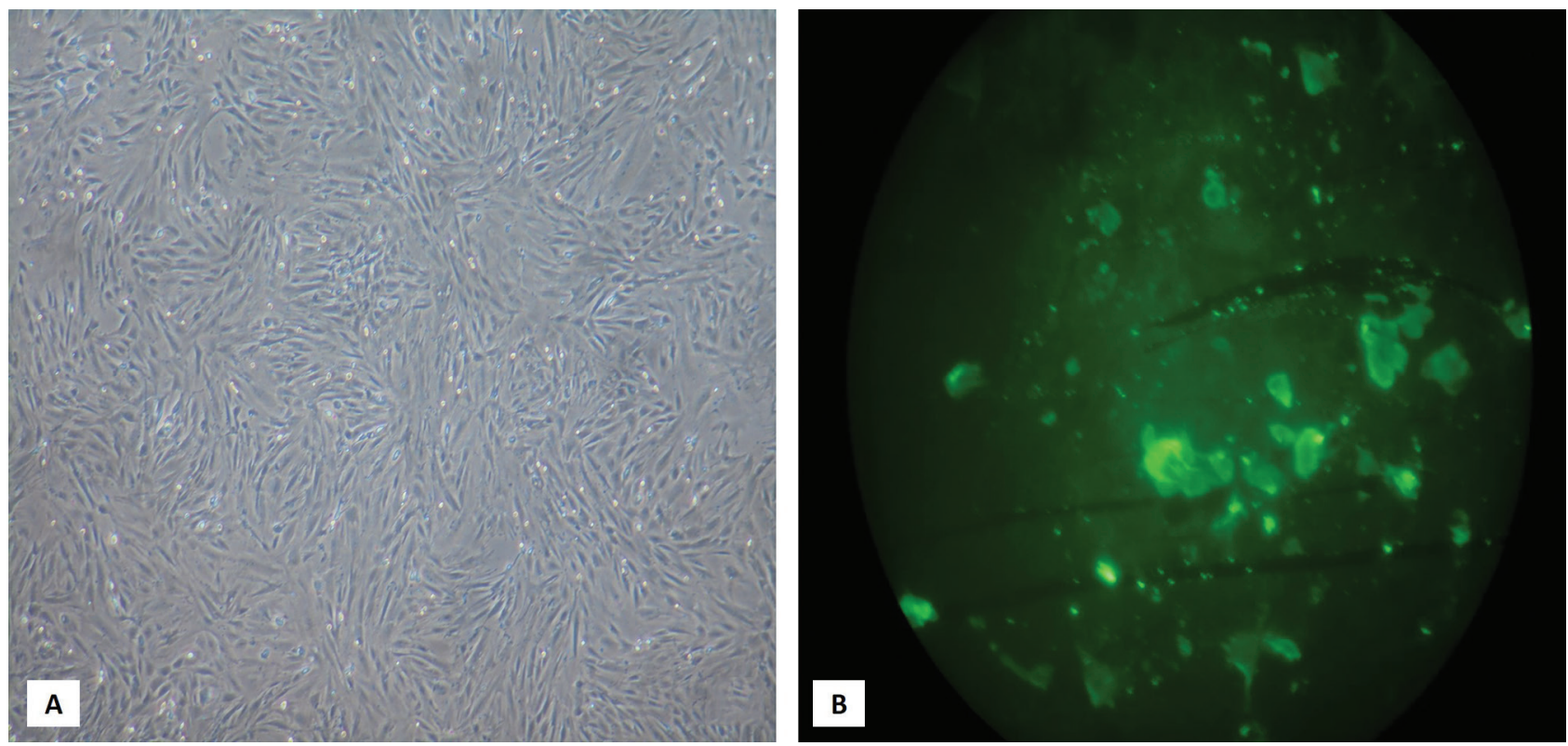

Fig. 1 Dental pulp stem cells culture on the 7th day, a fibroblastic-like cell appears attached to the base of the petri dish (A) and expresses CD105 (B).

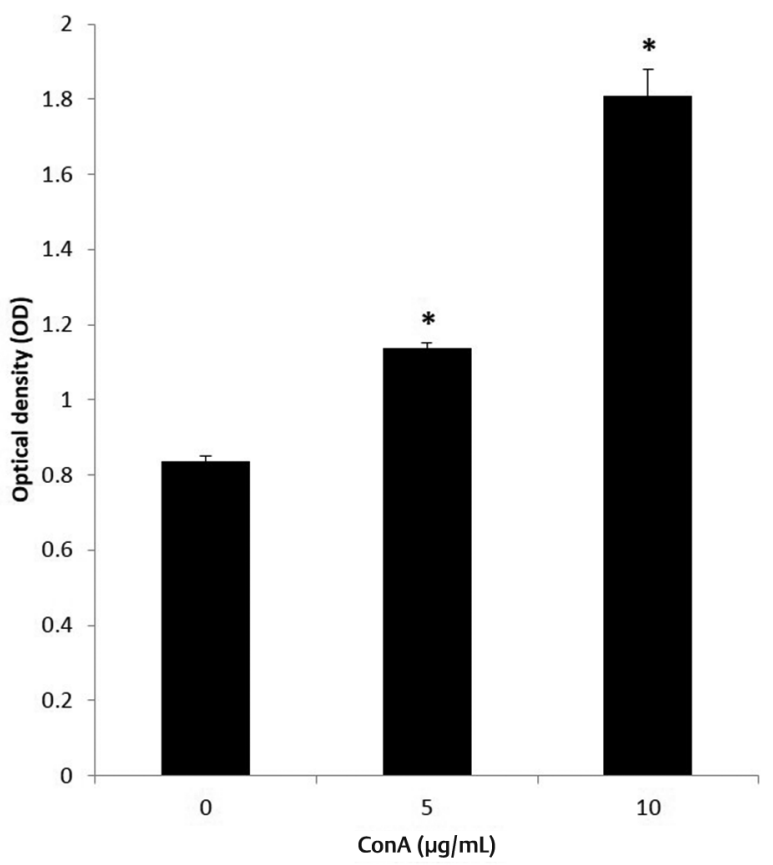

Fig. 2 ConA can increase the proliferation of DPSCs. DPSCs were cultured in osteogenic medium with 5 and $10 \mu \mathrm{g} / \mathrm{mL}$ ConA being added for 24 hours. An MTT assay was performed to measure cell proliferation. ConA at concentrations of 5 and $10 \mu \mathrm{g} / \mathrm{mL}$ significantly increased the proliferation of DPSCs. The data are mean \pm standard deviation for four cultures. ${ }^{*} p<0.05$ as compared with the control. ConA, concanavalin A; DPSC, dental pulp stem cell; MTT, 3-(4,5-dimethylthiazol-2-yl)-2, 5-diphenyltetrazolium bromide.

\section{DPSCs Osteogenic Differentiation Ability after the Addition of ConA}

To evaluate the effect of ConA on osteogenic differentiation of DPSCs, culture staining was performed with alizarin red on day
21. It was observed that the addition of 5 and $10 \mu \mathrm{g} / \mathrm{mL}$ of CoA increased the osteogenic differentiation of DPSCs characterized by mineralization. A concentration of $10 \mu \mathrm{g} / \mathrm{mL}$ produced higher osteogenic differentiation ability than $5 \mu \mathrm{g} / \mathrm{mL}$ ( - Fig. 3).

\section{Discussion}

Stem cells are a new alternative for regenerating pulp tissue. Dental pulp tissue is a very promising source of MSC in regenerative dentistry because it has multidifferentiation properties, isolation processes that are noninvasive and efficient, immunosuppressive properties and similarities with osteoblasts. ${ }^{16-18}$ DPSCs can differentiate into odontoblasts in vivo. DPSCs are capable of regenerating dental-pulp-like complex by forming reparative dentin-like structures on the surface of dentin..$^{2,5,19}$ For regeneration therapy using stem cells, a considerable number of cells are required. Because the number of stem cells in the body is very limited, a method to increase their number without eliminating their differentiation capabilities must be developed. In this study, ConA was used to improve the proliferation and osteogenic differentiation of DPSCs.

In this study, ConA can increase the proliferation of DPSCs in vitro. Lectins have been shown to exert a strong effect on the proliferation and differentiation of various animal cells, including lymphocytes, osteoblasts, and chondrocytes. . $^{10,11,20,21}$ ConA induces changes in the morphology and proliferation of lymphocyte cells. In chondrocyte cell culture, ConA induces changes from fibroblastic cells to spherical cells, while also increasing aggrecan synthesis within 24 hours. This effect is greater than those of growth factors and hormones. ${ }^{10,20}$ In the other hand, increased proliferation of DPSCs due to the addition of ConA does not occur in tumor cells. Lectins can inhibit proliferation and have a cytotoxic effect on tumor cells. ${ }^{22}$ Moreover, ConA induces apoptosis in human melanoma 

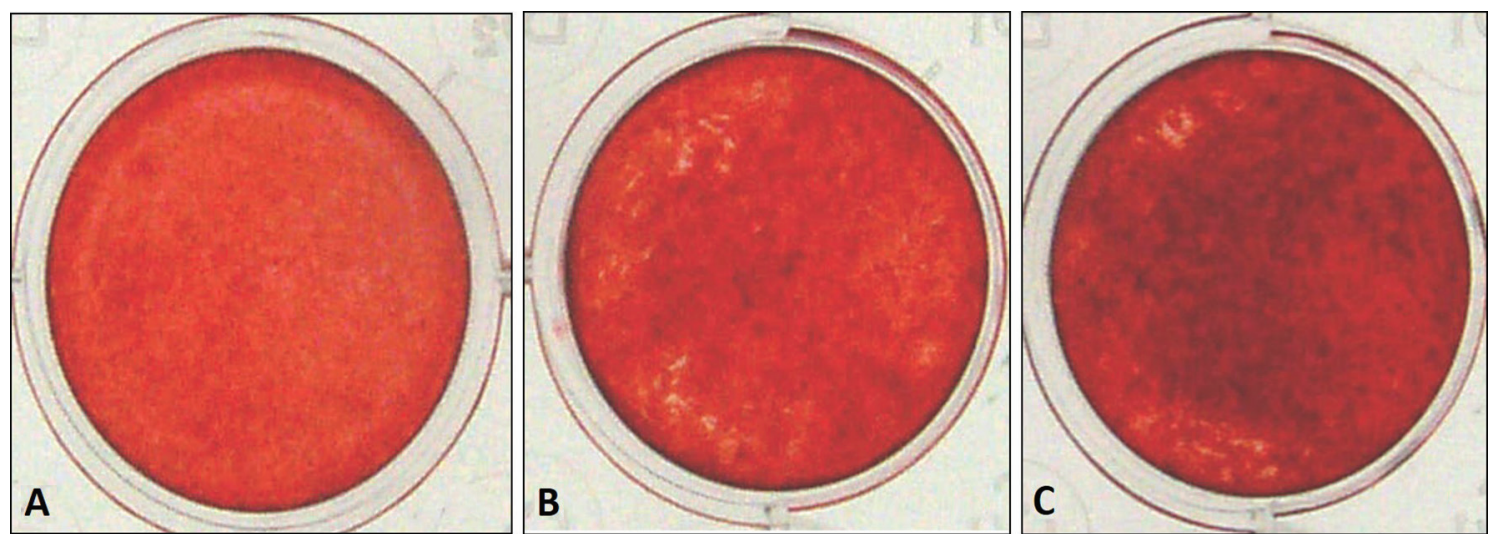

Fig. 3 ConA can increase osteogenic differentiation of DPSCs. DPSCs cultures are maintained in the osteogenic medium (A) or in osteogenic medium with 5 (B) and $10 \mu \mathrm{g} / \mathrm{mL}$ of ConA (C). The cell layers were stained with alizarin red on day 21. ConA, concanavalin A; DPSC, dental pulp stem cell.

cells. Subsequently, ConA caused mitochondrial transmembrane potential collapse, cytochrome $c$ release, activation of caspases, and eventually triggering a mitochondria-mediated apoptosis. $^{23}$

In this study, it was also found that ConA can increase mineralization in DPSCs cultures which are placed in an osteogenic medium. This finding is consistent with those of previous studies that the addition of ConA can improve the process of MSC mineralization or calcification..$^{12}$ The increase in osteogenic differentiation of DPSCs is probably due to ConA increasing BMP-2 levels in DPSCs culture. BMP-2 is an inductive growth factor for osteogenic differentiation of various stem cells. ${ }^{24}$ ConA can increase levels of osteocalcin and BMP-2 proteins in MSC culture. In addition, ConA increases ALP, Runx2, osteocalcin, BMP2, BMP4, and BMP-6 at the level of mRNA expression. ${ }^{12}$

\section{Conclusion}

From this study, it can be concluded that ConA can increase proliferation, maintain, and enhance osteogenic differentiation of DPSCs in vitro. Further studies should be performed to understand the signaling pathway underlying the effects of ConA on proliferation and osteogenic differentiation of DPSCs.

\section{Conflict of Interest}

None declared.

\section{References}

1 Ponnaiyan D, Jegadeesan V. Comparison of phenotype and differentiation marker gene expression profiles in human dental pulp and bone marrow mesenchymal stem cells. Eur J Dent 2014;8(3):307-313

2 Gronthos S, Mankani M, Brahim J, Robey PG, Shi S. Postnatal human dental pulp stem cells (DPSCs) in vitro and in vivo. Proc Natl Acad Sci U S A 2000;97(25):13625-13630

3 Arthur A, Rychkov G, Shi S, Koblar SA, Gronthos S. Adult human dental pulp stem cells differentiate toward functionally active neurons under appropriate environmental cues. Stem Cells 2008;26(7):1787-1795
4 Sugiyama M, Iohara K, Wakita $\mathrm{H}$, et al. Dental pulp-derived CD31- ${ }^{-}$CD146- side population stem/progenitor cells enhance recovery of focal cerebral ischemia in rats. Tissue Eng Part A 2011;17(9-10):1303-1311

5 Miura M, Gronthos S, Zhao M, et al. SHED: stem cells from human exfoliated deciduous teeth. Proc Natl Acad Sci USA 2003;100(10):5807-5812

6 Laino G, Graziano A, d'Aquino R, et al. An approachable human adult stem cell source for hard-tissue engineering. J Cell Physiol 2006;206(3):693-701

7 d'Aquino R, Graziano A, Sampaolesi M, et al. Human postnatal dental pulp cells co-differentiate into osteoblasts and endotheliocytes: a pivotal synergy leading to adult bone tissue formation. Cell Death Differ 2007;14(6):1162-1171

8 Simpson AH, Mills L, Noble B. The role of growth factors and related agents in accelerating fracture healing. J Bone Joint Surg Br 2006;88(6):701-705

9 Hanada K, Dennis JE, Caplan AI. Stimulatory effects of basic fibroblast growth factor and bone morphogenetic protein- 2 on osteogenic differentiation of rat bone marrow-derived mesenchymal stem cells. J Bone Miner Res 1997;12(10):1606-1614

10 Yan W, Pan H, Ishida $\mathrm{H}$, et al. Effects of concanavalin A on chondrocyte hypertrophy and matrix calcification. J Biol Chem 1997;272(12):7833-7840

11 Oda R, Suardita K, Fujimoto K, et al. Anti-membrane-bound transferrin-like protein antibodies induce cell-shape change and chondrocyte differentiation in the presence or absence of concanavalin A. J Cell Sci 2003;116(Pt 10) :2029-2038

12 Sekiya K, Nishimura M, Suehiro F, Nishimura H, Hamada T, Kato Y. Enhancement of osteogenesis by concanavalin A in human bone marrow mesenchymal stem cell cultures. Int J Artif Organs 2008;31(8):708-715

13 Hilkens P, Gervois P, Fanton Y, et al. Effect of isolation methodology on stem cell properties and multilineage differentiation potential of human dental pulp stem cells. Cell Tissue Res 2013;353(1):65-78

14 Dominici M, Le Blanc K, Mueller I, et al. Minimal criteria for defining multipotent mesenchymal stromal cells. The International Society for Cellular Therapy position statement. Cytotherapy 2006;8(4):315-317

15 Byun YK, Kim KH, Kim SH, et al. Effects of immunosuppressants, FK506 and cyclosporin A, on the osteogenic differentiation of rat mesenchymal stem cells. J Periodontal Implant Sci 2012;42(3):73-80

16 Bakopoulou A, Apatzidou D, Aggelidou E, et al. Isolation and prolonged expansion of oral mesenchymal stem cells under 
clinical-grade, GMP-compliant conditions differentially affects "stemness" properties. Stem Cell Res Ther 2017;8(1):247

17 Yang H, Li J, Sun J, et al. Cells isolated from cryopreserved dental follicle display similar characteristics to cryopreserved dental follicle cells. Cryobiology 2017;78:47-55

18 Rodas-Junco BA, Villicaña C. Dental pulp stem cells: current advances in isolation, expansion and preservation. Tissue Eng Regen Med 2017;14(4):333-347

19 Batouli S, Miura M, Brahim J, et al. Comparison of stem-cell-mediated osteogenesis and dentinogenesis. J Dent Res 2003;82(12):976-981

20 Yan WQ Nakashima K, Iwamoto M, Kato Y. Stimulation by concanavalin A of cartilage-matrix proteoglycan synthesis in chondrocyte cultures. J Biol Chem 1990;265(17):10125-10131
21 Nakamasu K, Kawamoto T, Shen M, et al. Membrane-bound transferrin-like protein (MTf): structure, evolution and selective expression during chondrogenic differentiation of mouse embryonic cells. Biochim Biophys Acta 1999;1447(2-3):258-264

22 De Mejía EG, Prisecaru VI. Lectins as bioactive plant proteins: a potential in cancer treatment. Crit Rev Food Sci Nutr 2005;45(6):425-445

23 Liu B, Li CY, Bian HJ, Min MW, Chen LF, Bao JK. Antiproliferative activity and apoptosis-inducing mechanism of Concanavalin A on human melanoma A375 cells. Arch Biochem Biophys 2009;482(1-2):1-6

24 Chen D, Zhao M, Mundy GR. Bone morphogenetic proteins. Growth Factors 2004;22(4):233-241 